\title{
GANIL, Present and Future
}

\author{
A.C.C. Villari \\ GANIL, B.P. 55027 Caen Cedex 5 France
}

(for the SPIRAL2 group)

Received on 29 October, 2003

\begin{abstract}
Based on the LINAG Phase 1 conceptual design, a two years detailed study on a ISOL-type facility for the production of high intensity exotic beams, named SPIRAL2, has been launched. The radioactive isotope beams are produced via the fission process, with the aim of $10^{13}$ fissions/s at least, induced in a $U C_{x}$ target either by fast neutrons from a $\mathrm{C}$ converter or by direct bombardment of fissile material. Fusion-evaporation residues, using heavy ions beams in different targets can also be produced in this facility. The driver, with an acceleration potential of $40 \mathrm{MV}$ will accelerate deuterons $(5 \mathrm{~mA})$ and $\mathrm{q} / \mathrm{A}=1 / 3$ ions $(1 \mathrm{~mA})$. Even heavier ions will be possible in a later stage. The driver consists in high-performance ECR sources, an RFQ cavity and independent phase superconducting resonators. As it is a linear accelerator, further upgrade will be possible in future.
\end{abstract}

\section{Introduction}

The systematic and very successful use of high-energy fragmentation at GANIL, (the first operational high intensity heavy ion accelerator in the 50-100 MeV/nucleon domain) for exploring the structure of nuclei far from stability, triggered the question of how to proceed even further in this domain. The study of nuclei far from stability has become one of the major activities at GANIL, and is one of its domains of excellence. It turns out from the principle of production and separation using a spectrograph the so-called in-flight method, which corresponds to the use of SISSI or LISE at GANIL that the optimum efficiency of the process is reached when the radioactive beam has a velocity similar to that of the primary beam. This production process, however, does imply losses in intensity and/or quality of the secondary beam, which become increasingly important as the beam is slowed down. The ISOL (Isotopic Separation OnLine) method, used at SPIRAL [1] since November 2001, provides the production and separation of radioactive ion beams, with subsequent acceleration by a $\mathrm{K}=265$ cyclotron CIME [2] between 1.7A and 25A MeV, thus allowing the study of nuclear reactions around the Coulomb barrier with radioactive ion beams. While SPIRAL is well suited for the production of light masses $(\mathrm{A}<80)$, the new project SPIRAL2 [3] will be devoted to the production of fission-like and fusion-evaporation radioactive ion beams, with subsequent acceleration by the CIME cyclotron. An important part of this project is the production of radioactive beams via fission induced by neutrons. The neutrons will be produced by the break-up of deuterons in a carbon wheel converter. The intensity of the deuteron beam ( $40 \mathrm{MeV}$ and 5 $\mathrm{mA}$ ) allows to reach $10^{13}$ fissions per second at least using a standard $U C_{x}$ target of density $2.3 \mathrm{~g} / \mathrm{cm}^{3}$. A possible increase of this yield can be obtained by changing the size or the density of the $U C_{x}$ of the target. If one considers the high density $U C_{2}$ with $11 \mathrm{~g} / \mathrm{cm}^{3}$, the yield will be enhanced by the same factor, i.e. a yield of $510^{13}$ fissions per second can be reached. The SPIRAL2 detailed design study will lasts up to November 2004 with IN2P3/CNRS, DSM/CEA and the Basse-Normandie Region support. In a second phase, the full accelerator LINAG (Linear Accelerator at GANIL) could allow to accelerate light and heavy ions to energies of around $100 \mathrm{~A} \mathrm{MeV}$ at intensities as high as $1 \mathrm{~mA}$, corresponding to an enhancement of the radioactive beam intensities of more than two orders of magnitude in comparison with the present GANIL accelerators.

\section{Physics with spiral2}

To achieve high intensities of RIBs, whilst reaching very far from stability regions, it is important to take advantage of various strategies in the production scheme. Therefore, modern next generation unstable ion beam facilities should consider all available techniques for the production of radioactive elements. Only a multi-beam heavy ion driver opens the possibility of adapting the best production method to the requested radioactive ion beam specifications. This is the main asset of the present GANIL laboratory, the unique facility offering both thin-target (In-flight) and thick-target (ISOL) methods. It is also the main characteristic of the present SPIRAL2 project, which will allow producing unstable beams by ISOL via the converter method or direct irradiation and by inflight technique. The combination of all these techniques opens new frontiers on the study of the nuclear chart. The main frontiers undertaken by SPIRAL2 are represented in the Fig. 1. The region (A) of the nuclear chart can be achieved by fission via direct bombardment of an uranium target with beams of $\mathrm{d},{ }^{3,4} \mathrm{He},{ }^{12,13} \mathrm{C}$ or heavier, while the region (B) represents the region which can be achieved via neutron induced uranium fission using 
deuteron beam in a carbon converter. In both regions, fundamental questions on nuclear structure and astrophysics are addressed. The region $(\mathrm{C})$ corresponds to a new totally unexplored region of the nuclear chart, which can be achieved via fusion-evaporation using unstable beams produced by SPIRAL2 in the regions (A) and (B). The region (D) corresponds to transfermium nuclei, which can be synthesised via fusion-evaporation with beams produced in the region (B). The region (E) corresponds to the superheavy island, which could be reached via fusion-evaporation of high intensity primary heavy ion beams on lead, bismuth or actinide targets. The region $(\mathrm{F})$, which is the $\mathrm{N}=\mathrm{Z}$ line in the so-called neutron deficient zone, can be achieved via fusionevaporation of light-heavy ions with selected targets. Both ISOL and in-flight techniques can be used for producing ions in this zone, allowing to produce high intensity beams or short living isotopes. The region $(\mathrm{G})$ corresponds to light exotic nuclei, which could be produced with incomparable high intensities with SPIRAL2, allowing, for example, to address studies concerning unbound nuclei, limits of stability and neutrino physics. Moreover, SPIRAL2 will also address non nuclear physics studies where the need of high intensity neutron fluxes, high intensity stable light ion beams and unstable nuclear beams are needed, such as material sciences, atomic, plasma and surface physics.

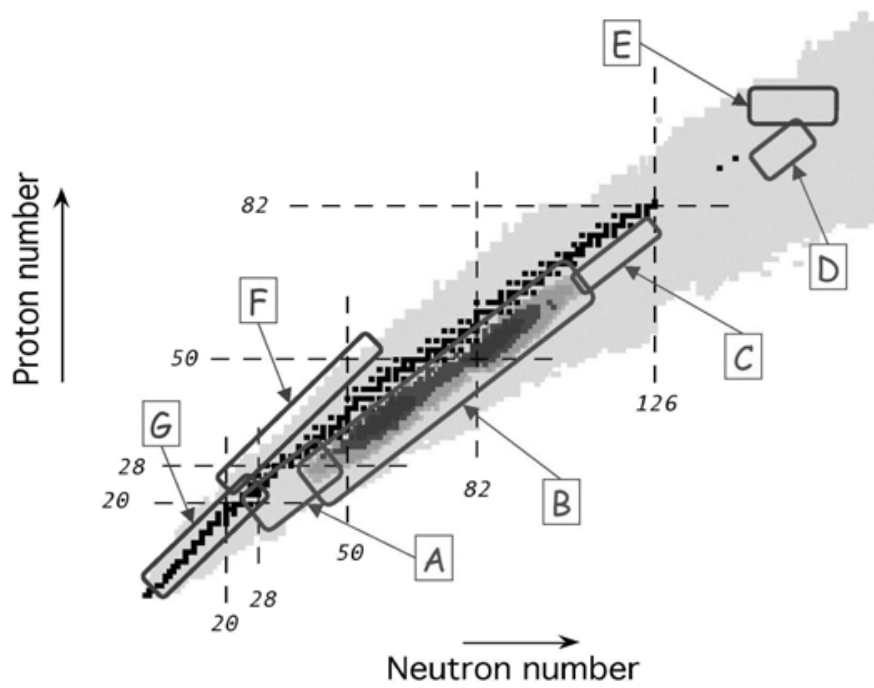

Figure 1. Main frontiers undertaken by SPIRAL2. See text.

\section{Production of fission fragment ra- dioactive beams}

One of the techniques proposed for SPIRAL2 has been already discussed in the EU-RTD report [4], consisting in the use of energetic neutrons to induce fission of ${ }^{238} \mathrm{U}$. The neutrons are generated by the break-up of deuterons in a thick target, the so-called converter, of sufficient thickness to prevent charged particles from escaping. The energetic forward-going neutrons impinge on a thick production target of fissionable material, i.e. Uranium carbide $U C_{x}$.
The resulting fission products accumulated in the target diffuse to the surface from which they evaporate, are ionised, mass-selected, eventually charge breeded and finally postaccelerated. This method has several advantages and was suggested by Gerald Nolen [5] in the framework of RIA. The material of the highly activated converter can be chosen to withstand the power of the beam without constraint concerning the diffusion of radioactive atoms. Moreover, the temperature of the converter does not affect the neutron flux. As projectiles, neutrons do not contribute to the heating of the target material directly neither the entrance window, which can be very thick and do not present any special security issue. Neutrons then bombard the target loosing energy only in useful nuclear interactions and having a high penetrating power, which allows very thick targets to be used.

\subsection{The target and ion source production sys- tem}

The target and ion source production system is placed just behind the rotating carbon converter. With $10^{13}$ fission per second, the total power produced in the $U C_{x}$ target is $310 \mathrm{~W}$. As mentioned above, the production target perceives no influence on the primary beam. The target temperature is completely controlled by an independent heating system, with a power of about $5 \mathrm{~kW}$.

\section{The production target}

Two possibilities have been considered in this study for the fissile targets. The first one of $U C_{x}$ with $2.3 \mathrm{~g} / \mathrm{cm}^{3}$, using the technology developed and used for many years at CERN ISOLDE [6]. Oxide of uranium is mixed with carbon powder in a small container, pressed and heated at $2300 \mathrm{~K}$ during approximately one day. The chips produced have generally around $1 \mathrm{~mm}$ thickness and a diameter of $20 \mathrm{~mm}$. Larger diameter do not seem to be a problem, but smaller thickness could be very difficult to produce. We assumed in our calculations of production yields a thickness of $1 \mathrm{~mm}$. Any development in order to reduce this value would be welcome. The second possibility is the use of high density $U C_{2}$ target $\left(11 \mathrm{~g} / \mathrm{cm}^{2}\right)$ already developed at Gatchina Russia. The high density $U C_{2}$ allows either to reduce considerably the size of the target or to increase by a factor of around 5 the yields for a constant geometry. Preliminary results show that the diffusion properties of high-density $U C_{2}$ are similar to the low-density. The geometry adopted in the simulations is not the optimum. The best would be to have a $U C_{x}$ targets of conical shape, with an angle of approximately of 30-40 degrees, as proposed in ref. [7]. In order to simplify the simulations, a simple cylindrical geometry has been adopted in all cases. Moreover, a reasonable size of the $U C_{x}$ target has been adopted.

\subsection{Production rates}

Yield calculations of fission fragments with the LAHET+MCNP+CINDER code for $5 \mathrm{~mA}$ deuteron beam of $40 \mathrm{MeV}$ energy in a carbon converter, followed by a $U C_{x}$ 
target have been performed. The expected radioactive beam intensities (after diffusion, effusion, ionisation and acceleration) are shown in the plots of Fig. 2 for some elements: Zn, $\mathrm{Kr}, \mathrm{Sr}, \mathrm{Sn} \mathrm{Sb}$ and Xe. The in-target production yields are those calculated using the $11 \mathrm{~g} / \mathrm{cm}^{3} U C_{2}$. The Arrhenius coefficients used in this calculation were supposed to be the same as for $\mathrm{C}$ and Ta, both tabulated in the literature. The assumed $1+$ and $1+/ \mathrm{N}+$ ionisation efficiencies are adopted as $90 \%(1+)$ and $12 \%(1+/ \mathrm{N}+)$ for $\mathrm{Kr}$ and $\mathrm{Xe}, 30 \%(1+)$ and $4 \%(1+/ \mathrm{N}+)$ for $\mathrm{Zn}, \mathrm{Sr}, \mathrm{Sn}, \mathrm{I}$ and $\mathrm{Cd}$. The assumed acceleration efficiency in the CIME cyclotron is $50 \%$.

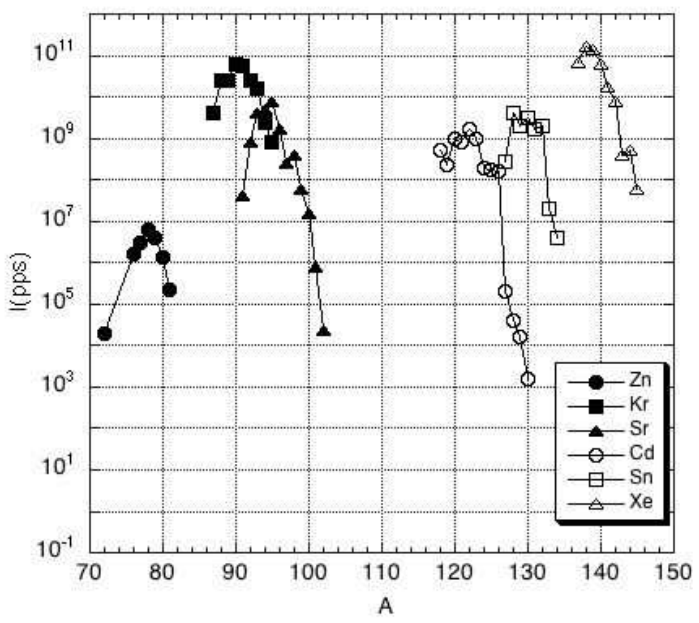

Figure 2. Expected radioactive beam intensities at SPIRAL2 after diffusion, effusion and acceleration for a list of selected elements.

\section{The driver accelerator}

The proposed LINAG1 driver for the SPIRAL2 project has the capability to accelerate a $5 \mathrm{~mA} \mathrm{~d}+$ beam up to 40 $\mathrm{MeV}$; nevertheless, the different parameters are optimised for $\mathrm{q} / \mathrm{A}=1 / 3$ ions up to $14.5 \mathrm{~A} \mathrm{MeV}$ in order to preserve a long term evolution towards an heavy ion driver. It is a continuous wave $(\mathrm{CW})$ mode machine, getting a maximum efficiency in the intensity transmission for heavy ions. It consists in an injector (ECR source + radio-frequency quadrupole), which accelerates the beam up to $0.75 \mathrm{~A} \mathrm{MeV}$, followed by a superconducting linear accelerator based on independently phased resonators.

\subsection{The RFQ injector}

The RFQ must operate in a CW mode. Its frequency has been chosen equal to $87.5 \mathrm{MHz}$, sub harmonic frequency of $350 \mathrm{MHz}$. This quite low value has been determined for the following reasons: - the RF power density is quite low at this frequency, and allows a solution based on a formed metal technology, leading to a cheap mechanical solution. - at lower frequency, the inter-vane distance is larger, and allows a higher margin for the mechanical tolerances. The RFQ output energy, $0.75 \mathrm{~A} \mathrm{MeV}$, has been preliminarily determined by the fact that the first cavities of the SC linac must allow a possible evolution of the machine for $\mathrm{q} / \mathrm{A}=1 / 5$ or 1/6 ions, which means that their beta value has to remain quite low (0.04). This value should already be optimised during the detailed study phase. Error simulations have been performed, considering mechanical tolerances of $+/-0.1 \mathrm{~mm}$ on the vanes machining and $+/-0.2 \mathrm{~mm}$ for misalignments. The results confirm that the deuteron beam transmission remains very close to $100 \%$. This gives a quite comfortable situation from the safety point of view: losses of $3 \%$ have been considered for the estimation of the protections.

\subsection{The superconducting linear accelerator}

The linac must have the capability to accelerate $d+$ and $\mathrm{q} / \mathrm{A}=1 / 3$ ions with the maximum energy gain, as well as to extend its performances to heavier ions in the future. A linac based on independent phase superconducting resonators is thus proposed. The Linac design requires accelerating voltages of the order of one MV/cavity and two beta values, around 0.07 and 0.14 , at a sub harmonic frequency of 350 $\mathrm{MHz}$ (availability of power sources). The frequency is 87.5 $\mathrm{MHz}$, not too high for the lowest beta cavity and not too low for the RFQ. Low beta super-conducting (SC) cavities in the beta range 0.04 to 0.2 are typically quarter wave resonators (QWR), operated at $4.2 \mathrm{~K}$ as the frequency is less than 500 $\mathrm{MHz}$. The beams dynamics calculations have been performed with the codes TraceWin, PARTRAN and LIONS. All these codes are able to use 3D field maps.

\section{Post acceleration}

The recently commissioned cyclotron CIME will perform the post acceleration in the SPIRAL2 project. It allows acceleration of heavy ions in the energy range of $1.7 \mathrm{~A} \mathrm{MeV}$ and $25 \mathrm{~A} \mathrm{MeV}$, depending on the q/A. For fission fragments and considering presently performances of the charge booster, optimal energies would be of the order of $8 \mathrm{~A} \mathrm{MeV}$.

\section{Summary}

In the present report we have described the technical aspects of the SPIRAL2 project and commented more precisely the aim of adding medium-mass nuclei to those available in the present GANIL. Fission induced by light particles $\left(\mathrm{d},{ }^{3} \mathrm{He}\right.$, ${ }^{4} \mathrm{He}$, etc.) was proposed to produce the radioactive ions, with an aim of $10^{13}$ fissions/s at least. We have shown here that the SPIRAL2 project can reach even higher fission rates using proven technologies. Using a C-converter and a $5 \mathrm{~mA}$ deuteron beam, neutron-induced fission will be $110^{13}$ fissions/s using standard-density $U C_{x}$, and $510^{13}$ fission/s for high-density $U C_{2}$. For both cases, a very small volume (240 $\mathrm{cm}^{3}$ ) ion source was selected, in order to have relatively fast 
diffusion effusion times for short-lived nuclei. In principle, larger volumes could result in even higher fission rates, up to $10^{14}$ fissions/s, for which the heat produced by fission in the ion source reaches $6 \mathrm{~kW}$, the present limit for the SPIRAL targets. The linear accelerator as a driver in this project belongs to the technology of high intensity accelerators, which are of strong current interest for various domains and is of rapid technological development. As a consequence of the high production rates, the radioprotection constraints become a major factor in the project. This implies a change of technology compared with SPIRAL, with higher costs for the target/ion-source and associated infrastructure. The technology of target plugs, as used at TRIUMF-ISAC [8], was chosen. It offers the guarantee of safe handling of the high levels of activity produced. The SPIRAL2 project is part of a multibeam policy of GANIL. Another aspect is its possible synergies with EURISOL [9]. We note that the most promising possibility examined for EURISOL for both driver and post-accelerator is a superconducting linac, precisely the same technology for the linac as proposed for the SPIRAL2 driver. These two machines could in fact be one and the same, by adding an appropriate RFQ for higher M/Q ratios. The proposed (final) LINAG driver should be able to accelerate ions up to mass 100 to energy of $100 \mathrm{~A} \mathrm{MeV}$. Even though the EURISOL proposal considers MW beam power, the experience with the $200 \mathrm{~kW}$ beams proposed for SPIRAL2 would be very relevant. A demonstrated competence in these areas would be a big advantage to any laboratory proposing to host such a facility.

\section{References}

[1] A.C.C. Villari, the SPIRAL group, Nucl. Instr. Meth. B204, 31 (2003).

[2] E. Baron, 14th Int. Conf. Cycl. Applic., Cape Town, South Africa (1995), Word Scientific.

[3] LINAG Phase I, a technical report, version 1.3, GANIL, June 27, 2002, GANIL R 0208.

[4] M.G. Saint Laurent et al., SPIRAL phase II European RTD report, GANIL R 01-03 2001.

[5] J. Nolen, A target concept for intense radioactive beams in the 132Sn region, Proc. Third Intern. Conf. On Radioactive Nuclear beams, ed. J. Morrissey, East lansing, Mi, May 24-27, 1993.

[6] P.V. Logatchev, L.B. Tecchio et al.; Graphite neutron target for exotic beams, SPES internal report, Legnaro 2000

[7] D. Ridikas, W. Mittig and A.C.C. Villari, Nucl. Phys. A701, 343c (2002).

[8] P.W. Schmor, Nucl. Phys. A171, 480c (2002).

[9] J. Vervier, Nucl. Instr. Meth. Phys. Res. B204, 759 (2003), The EURISOL web page: http://www.ganil.fr/eurisol/ $343 \mathrm{c}$. 